\title{
STRUCTURAL AND KINEMATIC PROPERTIES OF POPULATIONS OF THE ANDROMEDA GALAXY
}

\author{
J. EINASTO \\ W. Struve Astrophysical Observatory, Tartu, Estonia, U.S.S.R.
}

\begin{abstract}
New observational data (Spinrad, 1970; Van den Bergh, 1970; Rubin and Ford, 1970) are used to determine structural and kinematic parameters of the nucleus, the subsystem of globular clusters, and interstellar hydrogen in M31.

The mass derived for the nucleus from the new spectrophotometric data is in good agreement with the virial mass $6 \times 10^{8} \mathscr{M}_{\odot}$. Model calculations show that there is no appreciable exchange of stars between the nucleus and the bulge. The rotation energy of the nucleus is only $7.5 \%$ of the total kinetic energy; the central density is $2 \times 10^{6} \mathscr{M}_{\odot} \mathrm{pc}^{-3}$.

The mean radius of the subsystem of globular clusters is $4.5 \mathrm{kpc}$. This indicates that the subsystem of old stars is not identical with the spheroidal component of the galaxy, whose mean radius is only $1 \mathrm{kpc}$. Radial velocity dispersion of globular clusters is only half of that of the nucleus. This shows a strong dependence of the velocity dispersion on distance to the center of the galaxy and a bias in mass determination of a galaxy from velocity dispersion near the nucleus.

On the basis of data on rotation two mass distribution models have been found, differing from each other in respect of the mass concentration to the center. Spectrophotometric data on the stellar content of the bulge are urgently needed to solve the mass distribution problem.
\end{abstract}

\section{Introduction}

To construct a meaningful physical theory of the structure and evolution of a galaxy one needs reliable data on parameters, describing the spatial and kinematic structure of the galaxy and its subsystems of different ages. As such parameters one can adopt: the mass of the subsystem $\mathscr{M}$, its mean radius, $a_{0}$, the axial ratio of equidensity ellipsoids, $\varepsilon$ (supposing equidensity surfaces of subsystems to be ellipsoids of rotational symmetry and constant axial ratio), and suitable structural parameters determining the degree of concentration of the mass to the center of the system. As concentration parameters dimensionless quantities $K_{i}$ may be used (Einasto, 1968). In the present paper, however, we use for this purpose model-parameters $N, x_{0}$ of the modified exponential density law (Einasto, 1970).

In a series of papers (Einasto, 1969; Einasto and Rümmel, 1970a, b, c) we have studied the structure of the Andromeda galaxy and its subsystems and obtained preliminary values for descriptive parameters. Last year new observational data have become available for the nucleus, the subsystem of globular clusters, and interstellar hydrogen. It appears reasonable to use these data to redetermine structural parameters of the populations mentioned.

\section{Nucleus}

At the Basel IAU Symposium on Spiral Structure of the Galaxy we argued that different methods lead to different values for the mass of the nucleus of M31 (Einasto and Rümmel, 1970c). On the basis of photometric data (Redman and Shirley, 1937; Johnson, 1961; Kinman, 1965), and spectrophotometric data on the stellar content 
and mass-to-light ratio $\mathscr{M} / L=f=16$ (Spinrad, 1966) we obtained (Einasto, 1969) for the mass of the nucleus the value $\mathscr{M}=5 \times 10^{7} \mathscr{H}_{\odot}$. On the other hand, the known mean radial velocity dispersion of stars in the nucleus $\sigma_{r}=225 \mathrm{~km} \mathrm{~s}^{-1}$ (Minkowski, 1962), mean radius $a_{0}=5 \mathrm{pc}$ (obtained from the photometric profile of the nucleus), and axial ratio of equidensity ellipsoids $\varepsilon=0.8$ enable us to apply the tensor virial theorem. The resulting mass is $\mathscr{M}=5 \times 10^{8} \mathscr{M}_{\odot}$ (Einasto and Rümmel, 1970c).

The discrepancy in mass can be removed supposing, as Lynden-Bell (1969) does, that in the center of the galaxy a massive body exists. In this case the tensor virial theorem is to be modified. For the mean radial velocity dispersion of stars in the nucleus we have

$$
\bar{\sigma}_{r}^{2}=G a_{0}^{-1} \beta_{r}\left(\mathscr{M}_{c}+H_{0} \mathscr{M}_{0}\right)
$$

where $G$ is the gravitational constant,

$$
a_{0}^{-1}=\mathscr{H}_{0}^{-1} \int_{0}^{\infty} \mu(\alpha) \alpha^{-1} \mathrm{~d} \alpha
$$

the harmonic mean radius of the stellar subsystem of the nucleus, $\mathscr{M}_{c}, \mathscr{M}_{0}$ are the masses of the central body and the stellar population of the nucleus respectively, and $H_{0}, \beta_{r}$ dimensionless parameters. In formula $(2), \mu(\alpha)$ is the mass distribution function (mass of an ellipsoidal layer of equal density and unit equatorial thickness). The parameter $H_{0}$ depends only on the distribution of mass of the stellar population in the nucleus and is defined by the formula

$$
H_{0}=a_{0} \mathscr{H}_{0}^{-2} \int_{0}^{\infty} M(\alpha) \mu(\alpha) \alpha^{-1} \mathrm{~d} \alpha,
$$

where

$$
M(\alpha)=\int_{0}^{\alpha} \mu\left(\alpha^{\prime}\right) \mathrm{d} \alpha^{\prime}
$$

is the integral mass distribution function. The parameter $\beta_{r}$ depends on the axial ratio of equidensity ellipsoids $\varepsilon$ and on the inclination angle $i$ of the equatorial plane of the system to the line of sight

where

$$
\beta_{r}=\beta_{R} \cos ^{2} i+\beta_{z} \sin ^{2} i
$$

$$
\begin{aligned}
& \beta_{R}=1 / 2 e^{2}[(\arcsin e / e)-\varepsilon], \\
& \beta_{z}=\varepsilon^{2} / e^{2}[(1 / \varepsilon)-(\arcsin e / e)] \text { and } e^{2}=1-\varepsilon^{2} .
\end{aligned}
$$

The values of $H_{0}, \beta_{R}, \beta_{z}$ have been calculated for various models of stellar systems; the results will be published elsewhere. In particular for the nucleus of M31 we obtained from photometric observations $\varepsilon=0.8$, and $i=12.8$, which gives $\beta_{r}=0.375$. 
The space density of the stellar population can be fairly well represented by an exponential law, for which $H_{0}=0.312$. Adopting for the mean radius and the mass of the stellar component the values given above, we obtain for the central mass $\mathscr{M}_{c}=1.4 \times 10^{8} \mathscr{M}_{\odot}$.

After the Basel Symposium I requested Dr. H. Spinrad to determine the maximum value of mass-to-light ratio consistent with spectroscopic observations. Recently new data became available (Spinrad, 1970). According to a new model of stellar content the mass-to-light ratio increases to a value $f_{V}=45$, due to the necessity of adding faint $\mathrm{M}$ dwarfs. An upper limit to the number of red dwarfs is given by $\mathrm{V}-\mathrm{K}, \mathrm{V}-\mathrm{L}$ color observations (Sandage et al., 1969), which gives $f_{V} \leqslant 65$.

A re-examination of the photometric data mentioned above gives for the luminosity of the nucleus in visual light a value $L_{V}=1.42 \times 10^{7} L_{\odot}$. This is four times higher than our previous estimate. The difference is due to the absorption and color corrections: $A_{V}=0.6$ in our Galaxy, $A_{V}=0.3$ in M31 (Arp, 1965) and B - V =1.0 (Sandage et al., 1969). The luminosity profile in the central part of M31 is shown in Figure 1. Using these data we obtain for the mass of the stellar component of the nucleus $\mathscr{M}_{0}=$ $6.4 \pm 2.1 \times 10^{8} \mathscr{M}_{\odot}$. The mean error of the result is estimated allowing for uncertainties of $L_{V}$, and $f_{V}$.

On the other hand, applying a better model of the distribution of mass with $H_{0}=0.273$, we obtained from the virial theorem $\mathscr{M}_{0}=5.7 \pm 1.9 \times 10^{8} \mathscr{M}_{\odot}$. In this estimate we adopted $\mathscr{M}_{c}=0$; the sources of mean error are uncertainties in $\sigma_{r}$, and $a_{0}$.

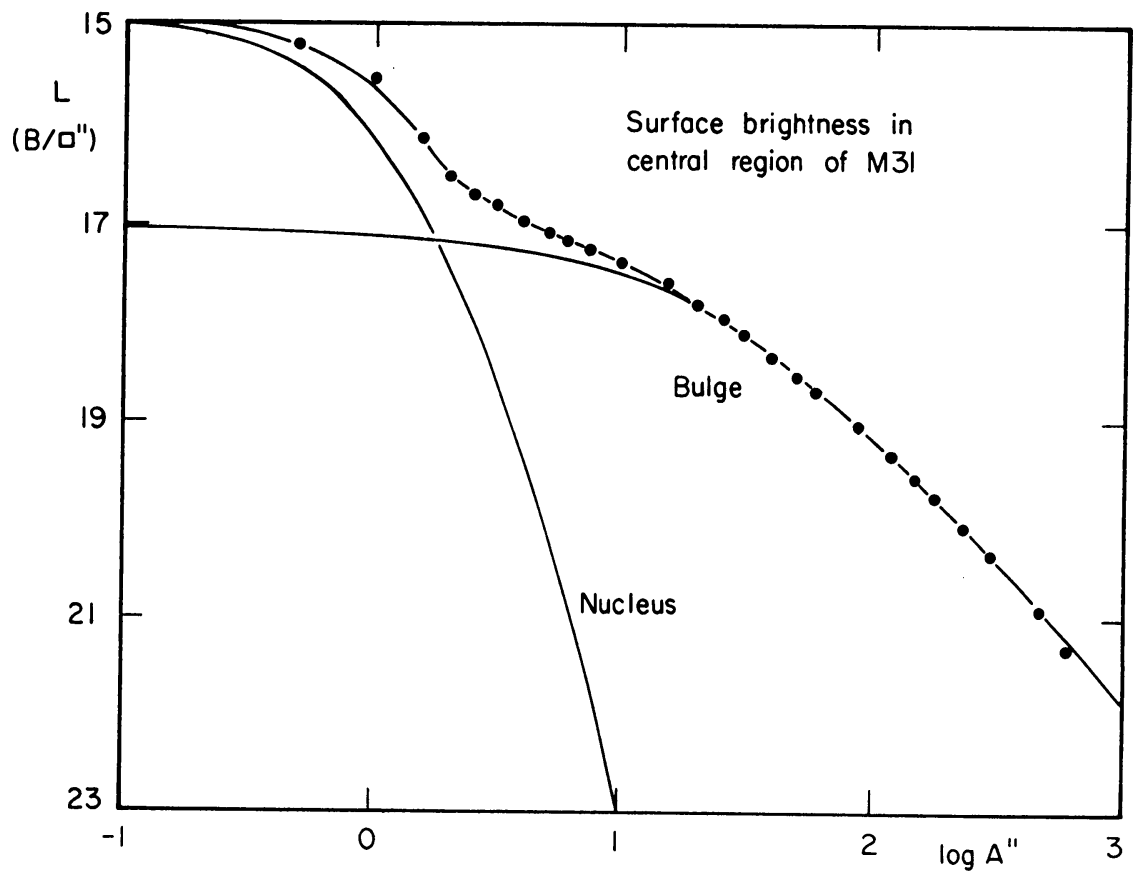

Fig. 1. Surface brightness of the nucleus and the bulge in the central region of M31. Dots represent observations, curves represent model profiles. 
Good agreement between these two independent estimates shows that it is not necessary to suppose the existence in the center of M31 of a body of large mass. However, ultraviolet observations carried out from the OAO indicate the presence of a UV-source in the nucleus of M31. If this source has dimensions small compared with the mean radius of the stellar component of the nucleus, formula (1) may be applied to estimate an upper limit for the point mass, which may be attributed to the UVsource. Assuming $\mathscr{M}_{0} \geqslant 4.0 \times 10^{8} \mathscr{M}_{\odot}$, we find $\mathscr{M}_{c} \leqslant 0.5 \times 10^{8} \mathscr{M}_{\odot}$.

Sandage et al. (1969) concluded from the U-B color variation along the major axis that the stellar content of the nucleus differs from that of the bulge. However, at present it is not clear whether the variation is due to the difference in stellar content or to the existence of a non-stellar UV-source in the center of M31. A difference in stellar content is not excluded because the nucleus is dynamically almost isolated and there is no appreciable exchange of stars between the nucleus and the bulge. To demonstrate this we have calculated, with the aid of a model of the mass distribution and the gravitational field of the nucleus, the apogalactic distances of stars moving through the center of the nucleus with various velocities (Table I). The majority

TABLE I

\begin{tabular}{rl}
\multicolumn{1}{l}{} & $R_{\text {apogal }}$ \\
\hline $\mathrm{km} \mathrm{s}^{-1}$ & $\mathrm{pc}$ \\
& \\
225 & 1.8 \\
450 & 8 \\
1080 & 85 \\
\end{tabular}

of the nucleus stars have velocities of some hundred $\mathrm{km} \mathrm{s}^{-1}$ and do not move far off from the center. Only stars having large velocities exceeding the escape velocity with respect to the nucleus, $1080 \mathrm{~km} \mathrm{~s}^{-1}$, go far away.

The mass density near the center of M31 according to our model is $2 \times 10^{6} \mathscr{M}_{\odot} \mathrm{pc}^{-3}$, the angular velocity of circular motion $186 \mathrm{~km} \mathrm{~s}^{-1} \mathrm{pc}^{-1}$. The mean observed angular velocity is $12 \mathrm{~km} \mathrm{~s}^{-1} \mathrm{pc}^{-1}$ (Lallemand et al., 1960). Using the tensor virial theorem and supposing a rigid-body rotation we find that the rotation energy is only $7.5 \%$ of the total kinetic energy of the nucleus. The binding energy of the nucleus (total negative energy per unit mass) is $7.5 \times 10^{4} \mathrm{~km}^{2} \mathrm{~s}^{-2}$.

\section{The Subsystem of Globular Clusters}

To reconstruct the past history of a galaxy it is important to know the structure and kinematics of populations of stars of various ages (cf. Einasto, 1970). As commonly accepted, the oldest stellar population belongs to the halo. Unfortunately the spatial density in the halo is very low and it is difficult to separate it by observation from bright disks and spirals. The study of elliptical galaxies shows that the transition from the halo to the bulge is a continuous one. Both populations have practically identical 
axial ratios of equidensity surfaces and can be described by the same density law of de Vaucouleurs' type. The mean radius of the spheroidal component of M31 (bulge and halo together) is (Einasto, 1969) $a_{0}=1 \mathrm{kpc}$.

In the case of globular clusters we have the possibility of studying the overall spatial distribution of an old population without any significant interference by other populations.

Our sample of globular clusters has been collected on the basis of Vetesinik's (1962) catalog. Photometric data on clusters were collected from various sources (Sharov, 1968). Probable open clusters are excluded from the general list; they lie in the V, B - V diagram to the left of the reddening line $A_{V} / E_{\mathrm{B}-\mathrm{V}}=2.5$ (Van den Bergh, 1970), going through the point $\mathrm{V}=18.0, \mathrm{~B}-\mathrm{V}=1.00 . H_{\alpha}$-regions (Haro, 1950), objects without any photometric data, and very faint clusters $(B>19.0)$, were also excluded. The remaining sample was divided into two groups: bright clusters $(B \leqslant 17.5)$ and faint clusters $(17.5<\mathrm{B} \leqslant 19.0)$, which consist of 101 and 92 clusters respectively.

On the basis of measured $(x, y)$ co-ordinates, published by Veteŝnik (1962), the galactocentric co-ordinates $W, U$ along the major and minor axes of the galaxy, and the projected distance from the center $A=\left(W^{2}+E^{-2} U^{2}\right)^{1 / 2}$ were calculated. $E$ is the apparent axial ratio of equidensity ellipses. We found $E=0.57$, which corresponds to the true axial ratio $\varepsilon=0.54$.

The distribution of clusters in $A$ is somewhat different in the two groups, which

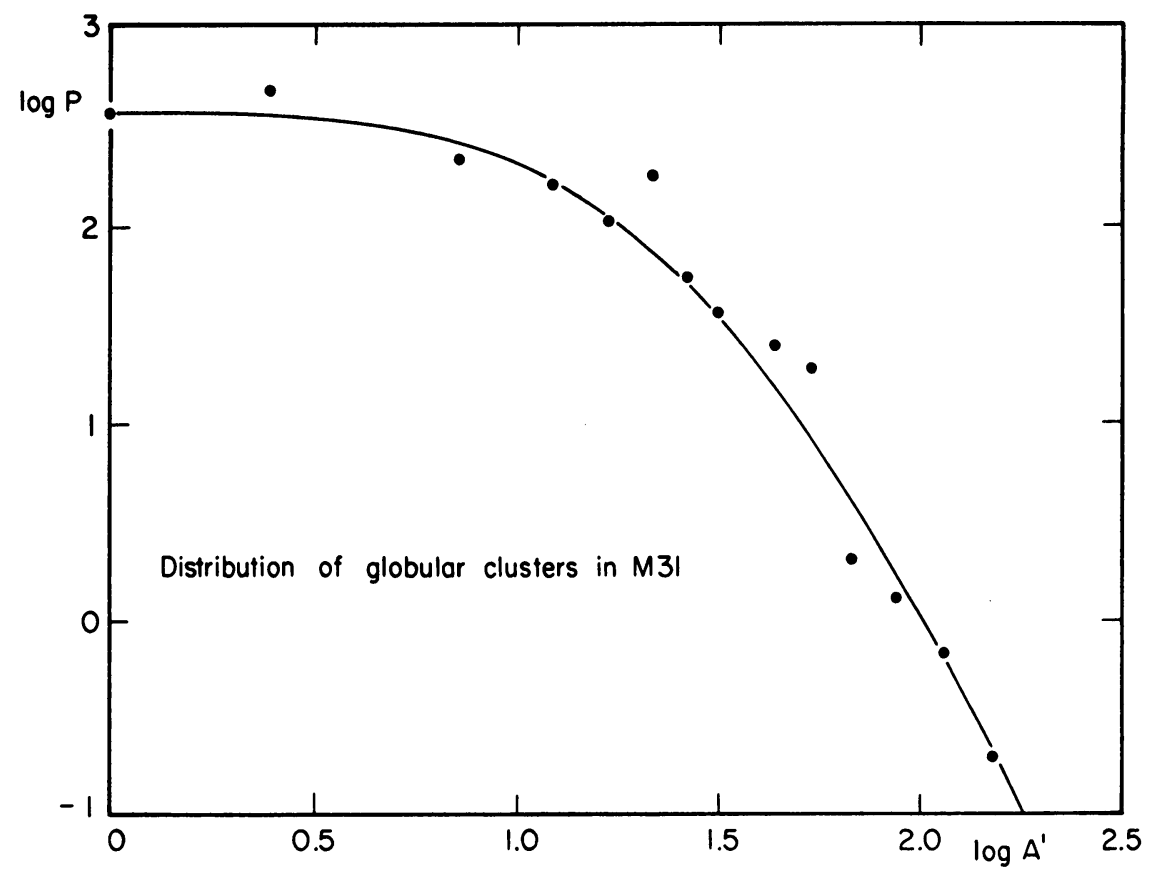

Fig. 2. Distribution of globular clusters in M31. As abscissae the logarithm of the major axes of equidensity ellipses (in minutes of arc) are plotted; as ordinates, the number of clusters per unit surface area (in arbitrary units). Dots represent observations; the solid curve represents the model. 
can perhaps be explained by a selection effect in observations. In the central and outer halo region the relative number of faint clusters is smaller. After a slight correction of numbers of faint clusters both groups were united and a general distribution was found (Figure 2). The distribution can be fairly well represented by a modified exponential density law (Einasto, 1970) with mean radius $a_{0}=4.5 \mathrm{kpc}, N=4$, and $x_{0}=10.5$.

This result shows that the system of globular clusters has a much greater mean radius than the spheroidal component of $M 31$. As we have no reason to believe that the spatial structure of the system of globular clusters differs significantly from that of the other old first generation stars, we come to the conclusion that the subsystem of old stars cannot be identified with the spheroidal component of the galaxy. In the central part of the galaxy star formation has probably taken place much longer than in the halo, giving rise to the formation of the bulge.

As to the kinematics of globular clusters, Van den Bergh (1970) has recently determined radial velocities for 44 bright globular clusters in M31. From his data the mean galactocentric radial velocity dispersion can be found. Adopting for the systemic velocity of the galaxy $-300 \mathrm{~km} \mathrm{~s}^{-1}$ (Van den Bergh, 1970; Rubin and Ford, 1970) we obtained

$$
\sigma_{r}=122 \mathrm{~km} \mathrm{~s}^{-1}
$$

which is only 0.54 of the mean radial velocity dispersion in the nucleus (Minkowski, 1962).

Preliminary model calculations show that the velocity dispersion of the subsystem of globular clusters depends mainly on the total mass of the galaxy and is quite insensitive to the mass concentration towards the center.

The difference between the mean velocity dispersions of the nucleus and the subsystem of globular clusters has one important consequence. Velocity dispersions near the nucleus have been used to determine the total mass of a galaxy. For example, by this method Brandt and Roosen (1963) obtained for M87 $\mathscr{H}=2.7 \times 10^{12} \mathscr{M}_{\odot}$, and $\mathscr{M} / L=85$. However, a strong dependence of the dispersion on the distance $R$ shows that the velocity dispersion near the center characterizes the mass and the size of the nucleus only. If the run of relative velocity dispersion in other galaxies is the same as in M31, the total mass of the galaxy and the respective mass-to-light ratio obtained from the virial theorem are approximately 4 times higher than the true ones.

\section{Interstellar Hydrogen}

The distribution and kinematics of interstellar hydrogen in M31 were analyzed on the basis of radio data in our report to the Basel Symposium (Einasto and Rümmel, 1970b). Recently new optical radial velocities of hydrogen clouds in M31 became available (Rubin and Ford, 1970). In the present paper the results of a preliminary study of these data are given.

In Figure 3 the velocities from Table I of the paper by Rubin and Ford are plotted 
as a function of $R$. If in one association several emission clouds have been observed, a weighted mean rotation velocity and distance have been used; points far from the major axis have been omitted. Hydrogen $21-\mathrm{cm}$ rotation velocities according to our treatment (Einasto and Rümmel, 1970b) are also indicated in the figure. The solid line represents the rotational curve adopted by Rubin and Ford (model 5 in Table III of Rubin and Ford's paper).

New observations confirm earlier results (Babcock, 1939) that the rotation velocity has a sharp maximum near $0.5 \mathrm{kpc}$ from the center and a deep minimum near $2 \mathrm{kpc}$. Identifying the rotation velocities with the circular ones, Rubin and Ford calculated a corresponding mass distribution model and found a negative projected density at about $2 \mathrm{kpc}$ from the center.

TABLE II

\begin{tabular}{lllllll}
\hline Quantity & Unit & Nucleus & Bulge & Halo & Disc & Flat \\
\hline$\varepsilon$ & & 0.80 & 0.60 & 0.54 & 0.10 & 0.02 \\
$a_{0}$ & $\mathrm{kpc}$ & 0.005 & 0.4 & 4.5 & 10 & 8 \\
$N$ & & 4 & 4 & 4 & 1 & 0.5 \\
$h$ & & 4.411 & 4.411 & 4.411 & 2.220 & 1.571 \\
$k$ & & $0.4165 \times 10^{-4}$ & $0.4165 \times 10^{-4}$ & $0.4165 \times 10^{-4}$ & 0.4245 & 1.128 \\
$L_{V}$ & $10^{9} L_{\odot}$ & 0.014 & 4.5 & 15.3 & 12.5 & 3.1 \\
$\mathscr{M}_{\text {I }}$ & $10^{9} \mathscr{M}_{\odot}$ & 0.6 & 19 & 62 & 126 & 10 \\
$\mathscr{M}_{\text {II }}$ & $10^{9} \mathscr{M}_{\odot}$ & 0.6 & 12 & 1 & 184 & 20 \\
$f_{\mathrm{I}}$ & & 42 & 4.2 & 4.1 & 10.1 & 3.2 \\
$f_{\text {II }}$ & & 42 & 2.7 & 0.07 & 14.7 & 6.5
\end{tabular}

However, it is difficult to adopt negative or very small positive projected densities, because photometric observations indicate no minimum in projected luminosity density in this region. A large negative gradient of circular velocity would imply instability of circular motion, also difficult to explain. Apparently low rotation velocity in this region is due to another cause.

Adopting a smooth distribution of mass and identifying maximum rotation velocities near $R=0.5 \mathrm{kpc}$ with the circular velocity, we can calculate parameters for a corresponding mass distribution model and find the run of circular velocity for the whole galaxy. The parameters of this model are given in Table II, the circular velocity in Figure 3 (Model II).

This model has no negative densities, but the mass-to-light ratios* of the bulge and the halo are improbably small, especially that of the halo. The model has also another essential deficiency. The observed velocity dispersion of stars in the central parts of M31 is comparable to the rotation velocity (see preceding section) and the corresponding term in the hydrodynamic equations cannot be neglected. This indicates that the circular velocity must be considerably greater than the rotational one.

* The luminosities given in Table II are converted into the $V$ system adopting $B-V=1.0$ for the nucleus, the bulge and the halo, and $\mathrm{B}-\mathrm{V}=0.4$ for the disc and the flat component, with a correction for mean absorption $A_{V}=1.0$. 


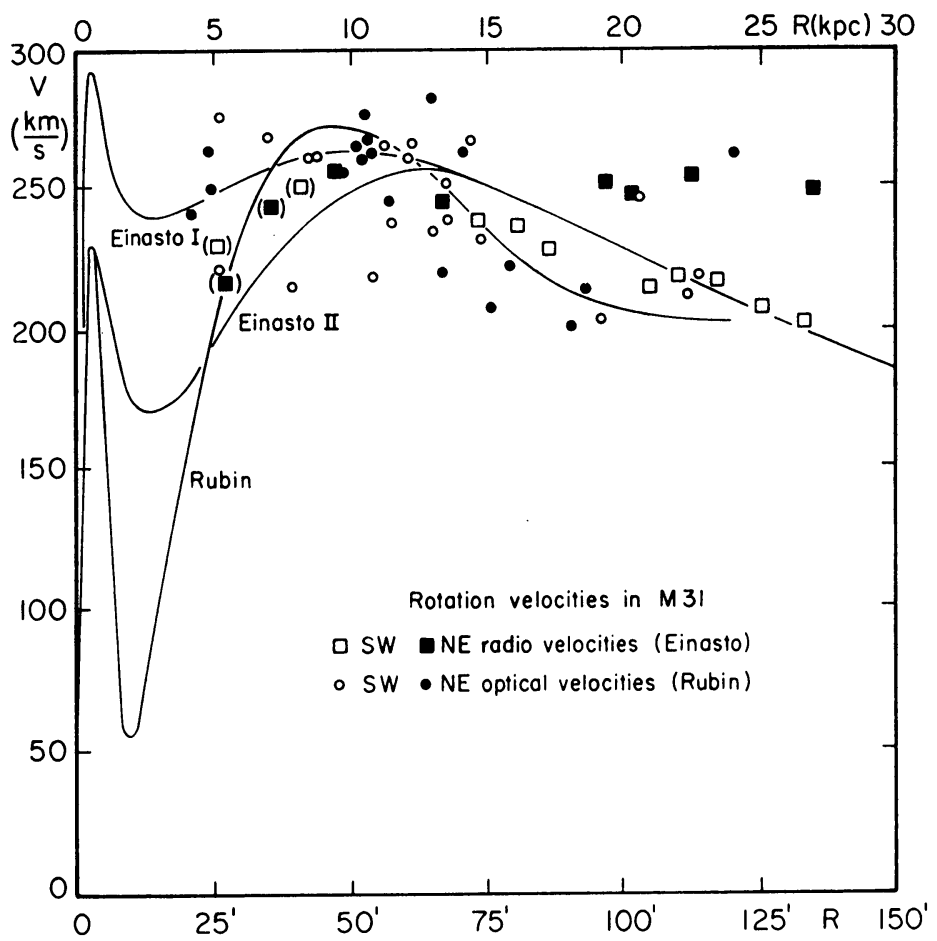

Fig. 3. Rotation of M31. Observed rotation velocities according to 21-cm radio data (Einasto and Rümmel, 1970b) and optical measurements (Rubin and Ford, 1970) are given with different signs. Circular velocity curves are shown by solid lines.

Both deficiencies are removed to a certain extent in another model whose parameters and circular velocity curve are also given in Table II and Figure 3 respectively (Model I). The mass-to-light ratios of the bulge and the halo seem to be still too low, compared with the mean mass-to-light ratio for the galaxy as a whole, $\mathscr{M} / L_{V}=6.1$. However, if we assume a considerably greater circular velocity in the central region, the velocity dispersion becomes improbably large.

To solve the problem of mass distribution in the central region of M31, spectrophotometric data on the stellar content of the bulge are urgently needed.

\section{Acknowledgements}

I am indebted to Dr H. Spinrad, Dr S. van den Bergh, and Mrs Dr V. Rubin for sending me the results of their work prior to publication, to Dr G. Kuzmin for suggestions connected with the use of the virial theorem, and to Mrs L. Einasto and U. Rümmel for programming the calculations and for help in the observational data processing.

\section{References}

Arp, H.: 1965, Astrophys. J. 141, 43.

Babcock, H. W.: 1939, Lick Obs. Bull. No. 498. 
Bergh, S. van den: 1970, Astrophys. J. Suppl. No. 171.

Brandt, J. C. and Roosen, R. G.: 1969, Astrophys. J. Letters 156, L59.

Einasto, J.: 1968, Tartu Publ. 36, 357.

Einasto, J.: 1969, Astrofizika 5, 137.

Einasto, J.: 1970, Tartu Teated No. 26, 1.

Einasto, J. and Rümmel, U.: 1970a, Astrofizika 6, 241.

Einasto, J. and Rümmel, U.: 1970b, in W. Becker and G. Contopoulos (eds.), 'The Spiral Structure of our Galaxy', IAU Symp. 38, 42.

Einasto, J. and Rümmel, U.: 1970c, in W. Becker and G. Contopoulos (eds.), 'The Spiral Structure of Our Galaxy', IAU Symp. 38, 51.

Haro, G.: 1950, Astron. J. 55, 66.

Johnson, H. M.: 1961, Astrophys. J. 133, 303.

Kinman, T. D.: 1965, Astrophys. J. 142, 1376.

Lallemand, A., Duchesne, M., and Walker, M. F.: 1960, Publ. Astron. Soc. Pacific 72, 76.

Lynden-Bell, D.: 1969, Nature 223, 690.

Minkowski, R.: 1962, in G. G. McVittie (ed.), Problems of Extra-Galactic Research, Macmillan Co., N.Y., p. 112.

Redman, R. O. and Shirley, E. G.: 1937, Monthly Notices Roy. Astron. Soc. 97, 416.

Rubin, V. C. and Ford, W. K., Jr.: 1970, Astrophys. J. 159, 379.

Sandage, A. R., Becklin, E. E., and Neugebauer, G.: 1969, Astrophys. J. 157, 55.

Sharov, A. S.: 1968, Astron. Zh. 45, 146.

Spinrad, H.: 1966, Publ. Astron. Soc. Pacific 78, 367.

Spinrad, H.: 1970 (private communication).

Veteŝnik, M.: 1962, Bull. Astron. Inst. Czech. 13, 180. 